$\xi=-1$

\title{
Network anomaly detection for protecting web services from the application layer bandwidth flooding attack
}

\author{
K V Raghavender ${ }^{1 *}$, Dr.P.Premchand ${ }^{2}$ \\ ${ }^{1}$ Research Scholor,Osmania University,Hyderabad \\ ${ }^{2}$ Professor, Dept of CSE, Osmania University, Hyderabad, T.S, India \\ *Corresponding author E-mail:kvraghu2011phd@gmail.com
}

\begin{abstract}
Web servers are generally situated in an efficient server center where these servers associate with the outside Web straightforwardly through spines. In the interim, the application layer Bandwidth flooding attack (ALBFA) assaults are basic dangers to the Web, especially to those business web servers. As of now, there are a few strategies intended to deal with the ALBFA assaults, however the greater part of them can't be utilized as a part of substantial spines. In this paper, we propound another technique namely BFADM to identify ALBFA assaults. Our work separates itself from past techniques by considering ALBFA assault discovery in overwhelming spine movement. Moreover, the recognition of ALBFA assaults is effortlessly deceived by streak swarm activity. Keeping in mind the end goal to beat this issue, our propounded technique develops a Constant Recurrence Vector and genuine opportune describes the movement as an arrangement of models. By looking at the entropy of ALBFA assaults and blaze swarms, these models can be utilized to perceive the genuine ALBFA assaults. We coordinate the above discovery standards into a modularized resistance design, which comprises of a headend sensor, an identification module and an activity channel. With a quick ALBFA discovery speed, the channel is equipped for letting the true blue demands through however the assault movement is ceased.
\end{abstract}

Keywords:ALBFA; Application-Laye; Distributed Denial of Service (DDoS); Popular Website.

\section{Introduction}

DoS assault is a pernicious endeavor to disturb the administration gave by systems or servers. The energy of a DoS assault is increased by fusing more than a large number of zombie machines through hoods [9] and mounting a DDoS assault. Utilizing botnets and rapid system advancements, current DoS assaults surpass the size of 300 Gbps turning into a noteworthy risk on the Web [10]. Being one of the most seasoned kind of assaults on the Web, DoS assaults are known for their troublesome behavior and capacity to drain the registering assets as well as transfer speed of their casualties in a matter of minutes. Albeit numerous guard components have been propounded to counter DDoS assaults [11], this still prevails to be a troublesome issue, particularly on the grounds that the assault activity tends to emulate typical movement as of late.

\section{Related work}

Alomari et al. [16] considered the risk of Botnet-based DDoS assaults starting at the application layer in light of the fact that these assaults made the income misfortunes for numerous businesses and government sites. The conceivable arrangements and the exploration headings for the future to determine application layer DDoS assaults were examined.

Gu and Liu [17] evaluated existing DoS assaults and key protection advancements in remote systems and portrayed system based and host based DoS assault procedures to delineate assault standards. DoS assaults were characterized by their real assault qualities. Current counterattack advances were additionally evaluated. DoS assaults and protections in 802.11 based remote systems were investigated at physical, Macintosh and system layers.

Zargar et al. [18] dissected the extent of the DDoS flooding assaults and ordered the assaults and accessible countermeasures in view of where and when these calculations could anticipate, identify, and react to the DDoS flooding assaults. Yau et al. [19] proposed another technique by utilizing level max-min reasonableness. A control-theoretic model was utilized for finding the union of calculation in light of various framework parameters. Distinctive models to speak to a decent client and assailant were utilized to clarify the circulations and conduct. The examination presumed that the throttle system is very compelling for forceful assailants in dropping aggressor activity over great client movement. The levelmax-min reasonableness gave preferable great client assurance over strategies proposed in the writing. Recreations were directed, and the outcomes demonstrated that switch throttling had low sending overhead in time and memory

The TCP layer is another principle combat zone for distinguishing DDoS assault. For instance, creators [20] mapped ICMP, UDP, and TCP bundle factual variations from the norm to particular DDoS assaults in light of MIB. Wang et al. [21] utilized the TCP SYN/Blade parcels for recognizing SYN flooding assaults. In [18] DDoS assaults were found by examining the TCP bundle header against the well-defined principles and conditions and recognized the contrast amongst ordinary and unusual activity. Noh et al. [22] endeavored to distinguish assaults by registering the proportion of TCP banners (including FIN, SYN, RST, PSH, ACK, and URG) to TCP bundles got at an Internet server.

\subsection{Existing}


Yi Xie et al aimingfor observing Web activity with a specific end goal to uncover dynamic moves in ordinary burst movement, which may flag the beginning of Application DDoS assaults amidst the glimmer swarm occasion. Our technique uncovers early assaults simply relying upon the report frame got from the server log. The propounded strategy depends on PCA, ICA, and HsMM. We directed the investigation with different avenues regarding diverse Application related DDoS assault modes (i.e., steady rate assaults, expanding rate assaults and stochastic beating assault) amidst a blaze swarm occasion gathered from a genuine follow. Our results related to simulation demonstrates that the framework could catch the move of Web activity caused by assaults under the glimmer swarm and the entropy of the watched information fitting to the HsMM can be utilized as the measure of variation from the norm. In our analyses, when the location edge of entropy is set 5.3, the DR is $90 \%$ and the FPR is $1 \%$. It additionally exhibits that the propounded engineering is required to be useful in observing Application DDoS assaults and in activating more committed location on casualty network.

\subsection{Motivations}

AL-DDoS assault recognition and malignant movement sifting systems have for quite some time been critical however troublesome issues to be tended to. Mainstream web servers are typically the ideal focuses for assailants to dispatch Application-DDoS assaults. With a specific end goal to secure web servers, analysts have propounded bunches of techniques to identify ApplicationDDoS assaults. Be that as it may, the vast majority of them have not met the prerequisites of location in the overwhelming movement condition. For instance, Yi Xie et al. received a shrouded semi-Markov procedure to show the conduct of Web clients $[1,3]$. The concealed semi-Markov approach is an intricate calculation. At the point when clients visit a site, it follows and records the entire history of every client. As indicated by our perception on two mainstream sites (Sina: www.sina.com and Taobao: www.taobao.com), the quantity of source IP locations may achieve a pinnacle of 104 solicitations for each second. It is observable that the concealed semi-Markov strategy is probably not going to perform successfully in spine activity. Another run of the mill approach against AL-DDoS assaults is to utilize CAPTCHA $[4,5]$. This technique expects clients to perceive strings in a fluffy picture and present a reaction to a web server for verification. In any case, clients here and there consider this operation as a negative affair to surf the Web. Paper [6] acquainted wavelets with recognize peculiarities in arrange movement. Be that as it may, wavelet investigation is for the most part a posthumous examination and can't be utilized for internet preparing. Paper [7] proposed a system to deliberately expand the transfer speed usage of honest to goodness clients. Be that as it may, this approach can't decrease the system blockage and the heap of web servers. Paper [8] proposed a countermeasure that comprised of a doubt task process and a DDoS-strong scheduler. The doubt procedure appoints a ceaseless 'esteemed versus double' measure onto every customer session. It additionally uses these qualities to decide whether and when to plan the solicitations of a session. Be that as it may, this approach is still too tedious to distinguish AL-DDoS assaults in huge volume movement.

In this specific paper, we were propelled to outline a barrier framework at the spine level. This framework can distinguish Application-DDoS assaults focusing on web servers. Right now, the vast majority of these web servers are sent together in a server farm associating straightforwardly to the spines. In this way, it is basic to execute a powerful strategy to recognize AL-DDoS assaults and channel the malignant activity in spines before they makes impairments the web servers. The propounded framework has low unpredictability and can genuinely execute in high volume of traffic.

\section{Proposed work}

BFADM is a mechanism for detection which is utilized against a few sorts of DDoS assaults mimicking streak swarms. It can recognize four sorts of irregular activity: rehashed ask for DDoS, recursive demand DDoS, rehashed workload DDoS and glimmer swarm. BFADM is partitioned into three distinct stages. The principal stage is the strange movement discovery, which is actualized in a front-end sensor. At the point when this stage distinguishes anomalous activity a "Consideration flag" is sent to the following stage, which is the DDoS assault recognition. At the point when the Consideration flag comes to the DDoS assault identification, this stage figures the recurrence of the approaching source IP address and its went by site and things. Along these lines an expected normal recurrence of the assets, for example, pictures and site pages on the site is computed. At a point, when the recurrence is figured, the entropy can be chosen which additionally is named as mess extent. The estimation of the entropy can figure out what sort of DDoS assault it is or on the off chance that it is a blaze swarm. The last stage is filtration. This will channel and expel the non-legitimate IP addresses while legitimate activity keeps on approaching the web server.

\subsection{Abnormal traffic detection}

The unusual movement discovery is the principal period of BFADM. The primary motivation behind this capacity is to identify sudden changes in HTTP GET asks for, i.e. inconsistency identification, sent to the front-end sensor. This capacity does not make any move if no oddities are recognized. In the event that strange data is identified from the approaching HTTP movement, a "Consideration" flag is sent to the following stage (DDoS assault recognition), which additionally investigates the information and settles on a choice. The movement obtained is utilized to recognize diverse sorts of utilization layer DDoS assaults and glimmer swarms. A few stages are taken before sending a Consideration flag, these are portrayed beneath.

The primary estimation is to dissect the approaching activity. This should be possible in a wide range of ways however BFADM predicts activity power by utilizing an Auto Relapse show (AR demonstrates). In relapse, past qualities affect future esteems, along these lines the AR show utilizes past watched activity to anticipate the difference in movement force later on. At first, the HTTP GET movement stream is observed. A period arrangement $\{y !, y !, \ldots, y !\}$ is shaped by the movement force which are examined in steady time interims. The activity force is ascertained in this theory "by the aggregate number of bundles got in a period interim". The movement force is anticipated from before perceptions with the assistance of the AR display. On the off chance that significant changes are identified, it can conceivably be an application-layer DDoS assault or a blaze swarm. The AR demonstrates that predicts the present intensity of the traffic or the movement is:

$$
\mathrm{Yt}=\sum \mathrm{k}=1 \text { to } n\left(\mathrm{at}^{\mathrm{k} *} \mathrm{x}_{\mathrm{t}-\mathrm{k}}\right)+\mathrm{e}_{\mathrm{t}}
$$

The variable $y \mathrm{t}$ is the expectation of $x \mathrm{t}$, which is the value of observation at a specific time $t$. The variable at $\mathrm{k}$ is a stationary model parameter, which implies that it doesn't differ when time changes, and $e !$ is the error value for observation. Also, at a specific time $\mathrm{t}$, the contrast between the perception $x \mathrm{t}$ and the expectation $y \mathrm{t}$ gives the remaining or model mistake $d \mathrm{t}$, which can be found in condition (2).

From that particular residual under time t, a standard deviation $\sigma \mathrm{d}$ 2 can be ascertained, as found in condition (3).

$$
d \mathrm{t}=|y \mathrm{t}-x \mathrm{t}|
$$

Thirdly the standard deviation $\sigma_{\mathrm{d}}^{2}$ : 
Presently, a limit found in condition (4) can be made for deciding whether the activity is irregular or not. In the event that $d$ ! is higher than $k \sigma$ !, unusual movement is recognized and a Consideration flag is sent to the DDoS assault recognition stage. In the inverse situation when no unusual activity is recognized, the strange movement identification stage sends a Reject flag to the DDoS assault recognition work which inactivates itself. The consistent alters the affectability of the limit and isn't set to a particular value.

$d \mathrm{t}>\sigma_{\mathrm{d}^{2}}(4)$

\subsection{DDoSattack detection}

The DDoS assault recognition is the second period of BFADM. At the point when a Consideration flag is received, the DDoS assault identification is initiated. By ascertaining the entropy or wreckage degree of the approaching activity, this capacity can choose what kind of DDoS assault happened, or if there is a blaze swarm. With a specific end goal to figure the entropy and recognize diverse assaults and blaze swarms, a few steps are taken.

To comprehend the significance of chaos degree/entropy, the definition is,

Assume there is a set $\{\mathrm{n}\}$, if the components in set $\{\mathrm{n}\}$ are situated dispersedly, at that point the Chaos Degree is higher. In case, if the components in set $\{\mathrm{n}\}$ are united in a few focuses by any composed shape, the Chaos Degree is lower and near 0". The scattering is comparable to what number of various assets being asked for, i.e. demands from various source IPs to various assets will demonstrate a higher wreckage degree calculation than a demand to one asset as it were.

BFADM can detect four types of abnormal traffic described below.

1) Repeated Request application layer DDoS attack: the focus is mostly on one or two resources on a specifically selected website.

2) Recursive Request application layer DDoS attack: the bots attack the same number of resources on several different web pages, which means that traffic is spread in different directions but continues to focus on the same resources at each attack.

3) Rehashed Workload application layer DDoS assault: this assault implements less bots yet hurts the site much more. Its principle objective is to always ask for large pictures and database search conclusion.

4) Streak swarm: is when immense number of legitimate clients visit a site.

Give whole number I a chance to indicate the stream ID, whole number $\mathrm{m}$ signify the maximum stream ID, $\mathrm{x} \_\mathrm{i}$ mean the quantity

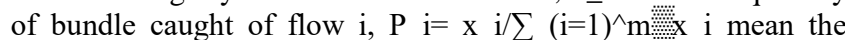
dissemination of probabilities of stream .

The entropy figured is:

$$
H(X)=-\sum_{i=1}^{m} P_{i^{*}} \log \left(P_{i}\right)(5)
$$

Average of $\mathrm{H}(\mathrm{X})$ is taken and is assigned as an ordinary Entropy H_n (X). To identify the assault, the entropy $\mathrm{H} \_\mathrm{C}(\mathrm{X})$ is computed in time window $\mathrm{T}$ consistently, signify $\mathrm{d}$ as supreme greatest deviation in Entropy H_C (X) from normal esteem H_n (X) while no assault is propelled. On the off chance that at time t, | H_C (X) $\mathrm{H} \_\mathrm{n}(\mathrm{X}) \mid>\mathrm{a} * \mathrm{~d}$, assault is valid. Here parameter a will be a composed parameter which decides the threshold value. A major an outcome in a wide threshold and low identification rate while a little an outcome in a restricted threshold and high false alert rate The most appropriate a through tedious tests, yet there is still opportunity to get better. The propelled entropy-based (AEB) scheme propounded in this paper is unrivaled in the accompanying three features:

To start with, distinguishing influxes of lawful movement Through our investigation, we have found that the bends shaped by a few sorts of LDOS assaults way to deal with the bend framed by legitimate activity, that implies just an extremely limited threshold controlled by a little a can let it know from typical movement, yet in the meantime a few influxes of lawful movement may likewise trigger caution. AEB gives a superior execution on this circumstance, getting higher identification rate and lower false caution rate.

Second, recognizing DDOS assaults from streak swarms there is a major distinction in the expanded and diminished speed of activity between them. In Streak swarms, all clients are difficult to get to all the while a similar server toward the start, on the grounds that the messages or news require set aside opportunity to spread among the clients; So the quantity of solicitations to the server is expanded continuously at that point to the pinnacle; correspondingly, toward the end phase of the glimmer swarms, all clients won't lose their fascinating to the server at the same time, so the quantity of solicitations to the server will be diminished steadily from the pinnacle.Be that as it may, in DDOS assaulting, the aggressors or zombies must dispatch an extensive number of solicitations to the server all the while or inside a brief span distinction to accomplish the coveted assault impact; Along these lines, the quantity of solicitations to the server is expanded strongly to achieve the pinnacle, and afterward will be diminished pointedly additionally toward the end phase of the DDOS assault. AEB ascertains entropy rate to recognize DDOS assaults from streak swarms.

Third, Web activity design fluctuates with time, as an outcome, H_n (X) may likewise change in a field. AEB change H_n (X) routinely to self-adjust network condition.

The substance of AEB is isolate and-vanquish methodology. We don't set the most appropriate limit controlled by a, rather, we isolate the entire field into various fields by various estimation of a. As exhibited in Fig. 1, the field is partitioned into 4 fields which are Typical Lv1, Lv2 and Lv3.

Characterize a_1, a_2, a_3, are composed conceivable estimations of an, and $0<\mathrm{a} \_1<\mathrm{a} \_2<\mathrm{a} \_3$.

Characterize $h$ as the component of $\mathrm{H} \_\mathrm{C}(\mathrm{X}), \forall \mathrm{h} \in \mathrm{H} \_\mathrm{C}(\mathrm{X})$,

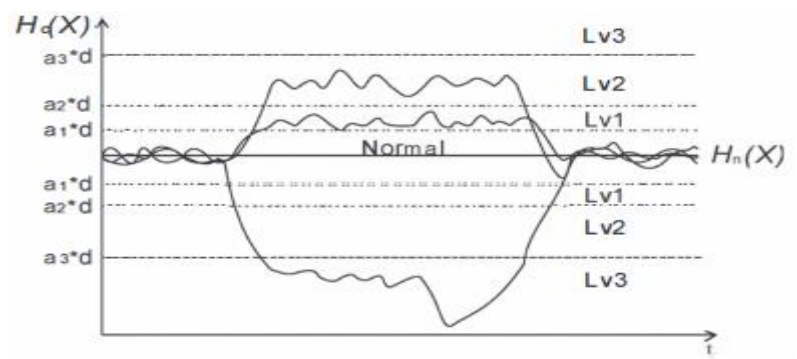

Fig. 1:Partition OfDDOS

If $\left|\mathrm{h}-\mathrm{H} \_\mathrm{n}(\mathrm{X})\right|<\mathrm{a} \_\mathrm{I}^{*} \mathrm{~d}$, at that point $\mathrm{h} \in$ Ordinary;

If $\mathrm{a} \_1 * \mathrm{~d}<\left|\mathrm{h}-\mathrm{H} \_\mathrm{n}(\mathrm{X})\right|<\mathrm{a} \_2 * \mathrm{~d}$, at that point $\mathrm{h} \in \mathrm{Lv} 1$;

If a_2 $* \mathrm{~d}<\left|\mathrm{h}-\mathrm{H} \_\mathrm{n}(\mathrm{X})\right|<\mathrm{a} \_3 * \mathrm{~d}$, at that point $\mathrm{h} \in \mathrm{Lv} 2$;

If $\left|h-H \_n(X)\right|>a \_3 * d$, at that point $h \in \mathrm{Lv} 3$;

The Typical field content a large portion of rushes of lawful traffic. At whatever point $\mathrm{H}_{-} \mathrm{C}(\mathrm{X}) \not \subset$ Normal, DDOS assaults may happen.

That prompts one of the accompanying three cases:

1) H_C (X) $\subset \mathrm{Lv} 3$, in light of the fact that it is a major deviation fromH_n $(\mathrm{X})$, we consider it as high rate DDOS assaults which will trigger caution quickly.

2) H_C $(\mathrm{X}) \subset \mathrm{Lv} 2$, it may be LDOS assaults or blaze swarms, which ought to be analyzed painstakingly to characterize. We consider it as LOOS assault and trigger alert if H_C (X) fulfills the accompanying two conditions:

1) The expansion rate or reduction rate of $\mathrm{H}_{-} \mathrm{C}(\mathrm{X})$ break the given limit $\mathrm{j}$.

2) H_C (X) $\subset$ Lv2 in the following $k * T$ seconds. $k$ is a planned parameter and $\mathrm{k}>0, \mathrm{~T}$ is the time window. 
Else, it ought to be a glimmer swarm.

3) H_C (X) $\subset$ Lv1, it may be LDOS or floods of legitimate movement. Since the vast majority of floods of legitimate movement fall into the typical field, the few waves fall into Lv1should be short, while LDOS pump activity continually to clog arrange. We consider it as LDOS assault and trigger alert if $\mathrm{H}_{-} \mathrm{C}(\mathrm{X})$ fulfills the accompanying two conditions:

1) The expansion rate or abatement rate of $H_{-} C(X)$ break the given limit $\mathrm{j}$.

2) H_C (X) $\not \subset$ Typical in the following $1 * T$ seconds, 1 is an outlined parameter and. $1>\mathrm{k}$. A little 1 will abbreviate the reaction time of $\mathrm{AEB}$ yet increment false caution rate.

Else if $\mathrm{H} \_\mathrm{C}(\mathrm{X}) \subset$ Typical in $\mathrm{m} * \mathrm{~T}$ seconds, $\mathrm{m}$ is an outlined parameter and $m>>1$, we in addition to the new $H_{-} n \llbracket(X) \rrbracket^{\wedge^{\prime}}$ and $\mathrm{d}^{\wedge}$ to the first ones relatively. As a result, the parcel changes, thus the fields.

$$
\begin{array}{r}
H_{-} n(X)=\beta^{*} H_{-n}(X)+(1-\beta)^{*}\left[H_{-}(X)-H_{-}(X)^{\prime}\right] \\
d=\gamma * d+(1-\gamma)^{*}\left(d-d^{\prime}\right) \\
0<\beta<1,0<\gamma<1 .
\end{array}
$$

Hence, AEB can identify high rate DDOS rapidly, recognize LDOS from streak swarms precisely, and channel floods of legitimate movement painstakingly and self-adjust organize condition. This makes a rush of guests known as glimmer swarms. Since legitimate clients as a rule generally takes enthusiasm for a particular webpage or resource the HTTP GET solicitations of glimmer swarms are spread. In request to process the movement continuously a recurrence vector called $R F V$ is made for each asset on the site, and also for each source IP address with the particular asset that is asked. There are $i=1$ to $m$ assets, for example, pictures and pages, in the site. This implies the recurrence for asset 1 in the site is FQ agm in the $R F V$, for asset 2 is FQ ag 2 until asset $m$ which is FQ ag $\mathrm{m}$. It can be found in the condition below

$$
\begin{aligned}
\mathrm{RFV}= & \left\{\mathrm{FQag}^{1}, \mathrm{FQag}^{2}, \ldots . \mathrm{FQag}^{\mathrm{m}}\right\} \quad(9) \\
& \mathrm{FQag}_{\mathrm{n}}=((1 / \mathrm{T} 2-\mathrm{T} 1)+(1 / \mathrm{T} 3-\mathrm{T} 2)+\ldots .+(1 / \mathrm{Tn}-\mathrm{Tn}-1)) / \mathrm{n}(10)
\end{aligned}
$$

The variable $n$ in above condition (6) demonstrates how often a source IP address asked for a particular asset. For recognizing whether it is a DDoS assault or a blaze swarm, the entropy for the anomalous movement is ascertained in condition (7) and after that analyzed in condition (8). The variable is the RFV of source IP addresses and is the URLs of the Website pages required by the aggressors and customers. In BFADM the extent of mess gives the circulation of the sources and the objectives $T$. BFADM intermittently computes the chaos degree of the irregular traffic.

The required formula for computing the entropy is:

$$
\text { EOYn }=\sum \mathrm{i}=1 \text { to } \mathrm{mFQag}_{\mathrm{n}}{ }^{\mathrm{i}} \log \left(\mathrm{FQag}_{\mathrm{n}}{ }^{\mathrm{i}}\right)
$$

By looking at the anomalous activity which is received from stage 1 , and deciding whether a specific sort of DDoS assault or glimmer swarm has happened, can be found in condition (8). The entropy esteems are numbered as: 1) Rehashed Ask for application layer DDoS assault, 2) Recursive Ask for application layer DDoS assault, 3) Rehashed Workload application layer DDoS assault and 4) Streak swarm.

$$
\text { EOY n(s)2/EOY n(t)2> EOY n(s)1/EOY n(t)1> EOY n(s)3/EOY }
$$$$
n(t) 3>\text { EOY } n(s) 3 / E O Y n(t) 3
$$

\subsection{Filter}

The last period of BFADM is the channel. In the wake of figuring the entropy from condition (8), if the estimation of the entropy for a particular source IP is demonstrated as a DDoS assault, the IP address is viewed as abnormal. As found in Figure 4, when irregular activity achieves the channel the odd IP address gets dropped and authentic IP delivers goes to the web server. This stage utilizes Blossom channel for figuring out which source IP delivers that will be dropped or preceded to the Internet server. An unfilled Sprout Channel is a bit cluster of $\mathrm{m}$ bits. All things are set to be 0 . There are additionally $\mathrm{k}$ diverse hash works, each of which maps or hashes a few components to one of the $m$ positions in an exhibit with a uniform arbitrary distribution. In our framework, the length of the bit exhibit $\mathrm{m}$ is 220 and two hash capacities are actualized inside $(\mathrm{k}=2)$. Assume the IP addresses are portrayed by the spotted decimal documentation 'X.Y.Z.F', at that point the hash capacities are:

$\left(\mathrm{X}^{3}+\mathrm{Y}^{3}+\mathrm{Z}^{3}+\mathrm{F}^{3)} \operatorname{Mod} 2^{20}\right.$

\section{$(\mathrm{X} * \mathrm{Y} * \mathrm{Z} * \mathrm{~F}) \operatorname{Mod} 220$}

So as to include a component, we have to pass the IP deliver to each of the two hash works and get two exhibit positions. Set the bits at all these positions to 1 . To question for a component (test whether the IP address is in the set), we likewise pass it to each of the two hash capacities to get two positions. On the off chance, that any of the bits at these positions is equivalent to 0 , the IP address does not exist in the set. As we have found in our examination, it is fit for constraining the contention beneath $16 \times 10-4$. On the off chance, that all the hash capacities come back to 1 , either the IP address is in the set, or the bits have been set to 1 by embedding other IP addresses. The last circumstance will be considered as an impact. We set the channel which has two hash capacities and the length of the hash table is 220 . The evaluated amount of noxious IP addresses is 20,000. At that point, the likelihood of plot can be figured as in

POC $=$ limit $m>>n\left[1-(1-n / m)^{k}\right]^{k}$

Limit $\mathrm{m}>>\mathrm{n}\left(1-\mathrm{e}^{-(\mathrm{kn} / \mathrm{m})}\right)^{\mathrm{k}}<-(\mathrm{kn} / \mathrm{m}) \mathrm{k}<\left(\left(2 * 2 * 10^{\wedge} 4\right) /\left(10^{\wedge} 6\right)\right)^{\wedge} 2$

$=16 /\left(10^{\wedge} 4\right)$

The outcome implies that the quantity of $2^{20}$ bits involves $128 \mathrm{kB}$ memory for two hash tables. This memory cost is paltry for most current machines.

\section{Simulation results}

We compare our proposed system with BFADM and HSMM by varying number of Attacking nodes inside the network.

\subsection{Scenario description}

The simulation is carried out using Network Simulator (NS-2) and analysis is presented below. We evaluate the performance and validate the effectiveness of proposed BFADM through this simulation. The simulation environment, performance metrics and simulation results are presented in this section. We created our topology using GT-ITM topology generator and it includes 250 client nodes. and it has three level hierarchy which contains transit domain averagely, stub domain and nodes .we take two transit domains which had five nodes and each transit nodes connect to five stub domain averagely and each stub domains has twenty nodes average and $10 \mathrm{mpd}$ link for domain, $5 \mathrm{mbp}$ for stub domain and $2 \mathrm{mps}$ for nodes. Attack can be cover 10 percentage of the nodes except the 10 transit nodes which is 25 nodes are attack nodes. we assume that assault nodes will send some request segment of normal surfers and replay hot pages for entering ddos attack to the web server shich is used by victim. A comparative study on the metrics, with existing protocol namely HSMM are also presented in the graphs below. 


\subsection{Performance evaluation}

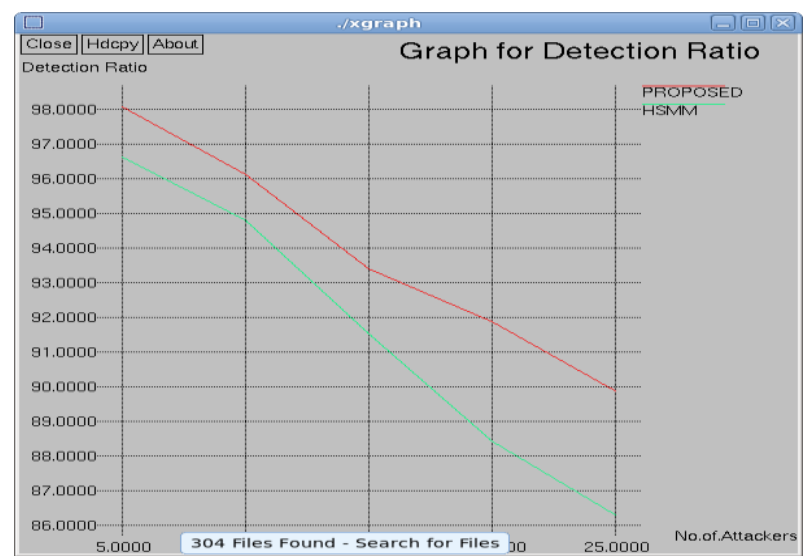

Fig. 2:Number of DDOS Attacker's vs.Detection Ratio.

Fig 2 shows performance between HSMM and our proposed work .In this graph by varying the number of attackers we plot the graph for detection ratio. detection ratio meanstotal number of attackers inside the network with how many attackers detected from them so by increasing attackers the detection ratio decreases but while comparing to existing HSMM our proposed detection ratio is higher about $6 \%$.

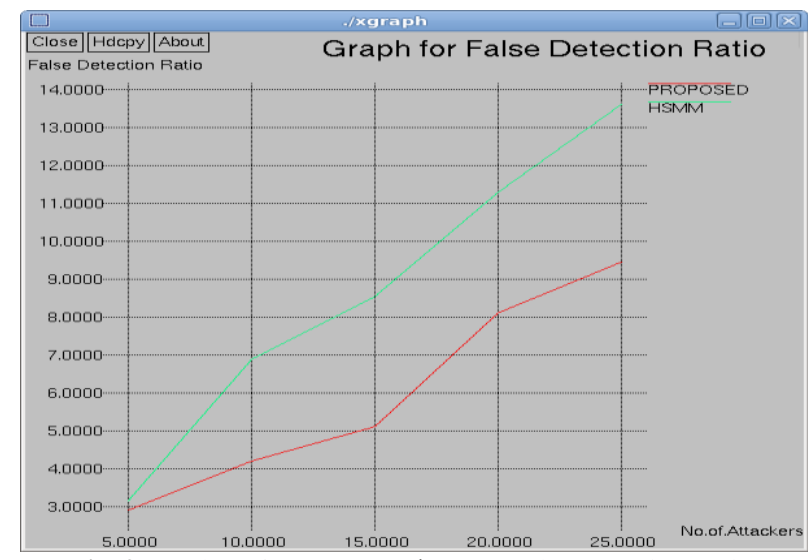

Fig. 3:Number of DDOS Attacker's vs.False Detection Ratio.

Fig 3 shows performance between HSMM and our proposed work .in this graph by varying the number of attackers we plot the graph for false detection ratio. False detection ratio means number of true nodes detected as attackers and attackers consider a true node .so by increasing attackers the false detection ratio increases but while comparing to existing HSMM our proposed detection ratio is lower about $8 \%$.

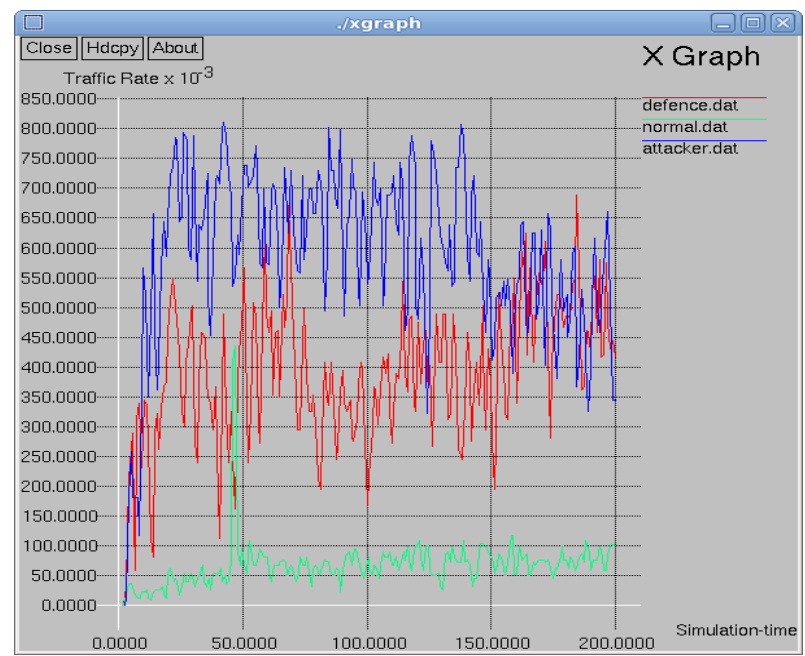

Fig. 4: Traffic Rate vs. Time.
Fig 4 shows performance between simulation time and traffic rate in normal behavior, when attacker enters in network and after defending the attackers .here we can see that the traffic rate is not disturbed when it is without attacker(indicated as green color) in network then when attacker enters into the network the traffic rate is increasing manner not consistent indicated as (blue color) and then after defense we control the traffic rate indicated as defense(red color).

\section{Conclusion}

So as to make protections for assaults it is important to acquire convenient and huge data by observing dynamic system exercises. The majority of the ebb and flow endeavors and inquires about spotlights on identifying system layer DDoS assault additionally called Net-DDoS assaults with stable foundation movement. This paper goes for flagging the Application Layer DDoS assaults amid streak swarm occasion. This is finished by uncovering the dynamic moves in typical burst activity and in this way checking Web activity. Our propounded technique develops a Constant Recurrence Vector and genuine auspicious portrays the movement as an arrangement of models. By inspecting the entropy of ALBFA assaults and glimmer swarms, these models can be utilized to perceive the genuine ALBFA assaults. We coordinate the above recognition standards into a modularized guard engineering, which comprises of a head-end sensor, a location module and a movement channel. With a quick ALBFA recognition speed, the channel is equipped for letting the true blue demands through yet the assault movement is halted.

\section{Acknowledgement}

Thankful to faculty members in Osmania University India and for providing resources, good guidance and environment.

\section{References}

[1] Y. Xie, S. Zheng Yu, Monitoring the application-layer ddos attacks for popular websites, IEEE/ACM Trans. Netw. 17 (1) (2009) 1525.https://doi.org/10.1109/TNET.2008.925628.

[2] Arbor. Networks, Worldwide network infrastructure security report, Tech. Rep., Arbor Networks, 2011.

[3] Y. Xie, S. Zheng Yu, A large-scale hidden semi-Markov model for anomaly detection on user browsing behaviors, IEEE/ACM Trans. Netw. $17 \quad$ (1) 65.https://doi.org/10.1109/TNET.2008.923716.

[4] L. von Ahn, M. Blum, N.J. Hopper, J. Langford, Captcha: using hard ai problems for security, in: EUROCRYPT, 2003, pp. 294 311.

[5] S. Kandula, D. Katabi, M. Jacob, A. Berger, Botz-4-sale: surviving organized ddos attacks that mimic flash crowds, in: Proceedings of the 2nd Conference on Symposium on Networked Systems Design and Implementation, NSDI'05, USENIX Association, Berkeley, CA, USA, 2005, pp. 287-300.

[6] P. Barford, J. Kline, D. Plonka, A. Ron, A signal analysis of network traffic anomalies, in: Proceedings of the 2nd ACM SIGCOMM Workshop on Internet Measurement, IMW '02, ACM, New York, NY, USA, 2002, pp. 7182.https://doi.org/10.1145/637201.637210.

[7] M. Walfish, M. Vutukuru, H. Balakrishnan, D. Karger, S. Shenker, Ddos defense by offense, ACM Trans. Comput. Syst. 28 (1) (2010) 1-54.https://doi.org/10.1145/1731060.1731063.

[8] S. Ranjan, R. Swaminathan, M. Uysal, E. Knightly, Ddos-resilient scheduling to counter application layer attacks under imperfect detection, in: Proceedings. INFOCOM 2006. 25th IEEE International Conference on Computer Communications, 2006, pp. 113.https://doi.org/10.1109/INFOCOM.2006.127.

[9] D. Dagon, G. Gu, C. P. Lee, W. Lee, "A Taxonomy of Botnet Structures," in Proc. of Annual Computer Security Applications Conference (ACSAC), Dec. 2007. https://doi.org/10.1109/ACSAC.2007.44.

[10] www.arbornetworks.com. 
[11] T. Peng, C. Leckie, K. Ramamohanarao, "Survey of NetworkBased Defense Mechanisms Countering the DoS and DDoS Problems," ACM Computing Surveys, vol. 39, no. 1, pp. 1-42, Apr. 2007. https://doi.org/10.1145/1216370.1216373.

[12] S. Kandula, D. Katabi, M. Jacob, A. W. Berger, "Botz-4-sale: surviving organized DDoS attacks that mimic flash crowds," in Proc. of NSDI, Boston, MA, 2005.

[13] C. Estan, G. Varghese, "New Directions in Traffic Measurement and Accounting," in Proc. of ACM SIGCOMM, Aug. 2002.

[14] R.R. Kompella, S. Singh, G. Varghese, "On Scalable Attack Detection in the Network," in Proc. of ACM Internet Measurement Conference (IMC), Oct. 2004 https://doi.org/10.1145/1028788.1028812.

[15] Z. Zhu, G. Lu, Y. Chen, Z. J. Fu, P. Roberts, K. Han, "Botnet Research Survey," in Proc. of IEEE COMPSAC, pp. 967-972, 2008. https://doi.org/10.1109/COMPSAC.2008.205.

[16] Alomari, Esraa, et al. "Botnet-based distributed denial of service (DDoS) attacks on web servers: classification and art." arXiv preprint arXiv: 1208. 0403 2012, pp. 24-32.

[17] Gu, Q., \& Liu, P. Denial of service attacks. Handbook of Compute Networks: Distributed Networks, Network Planning, Control, Management, and New Trends and Applications, Volume 3, 2007, pp. 454-468.

[18] Zargar, SamanTaghavi, James Joshi, and David Tipper. "A survey of defense mechanisms against distributed denial of service (DDoS) flooding attacks." Communications Surveys \& Tutorials, IEEE 15.4 2013, pp. 2046-2069. https://doi.org/10.1109/SURV.2013.031413.00127.

[19] Yau, David KY, et al. "Defending against distributed denial-ofservice attacks with max-min fair server-centric router throttles." IEEE/ACM Transactions on Networking (TON) 13.12005 , pp. 29 42

[20] J. B. D. Cabrera, L. Lewis, X. Qin, W. Lee, R. K. Prasanth, B. Ravichandran, and R. K. Mehra, "Proactive detection of distributed denial of service attacks using MIB traffic variables a feasibility study," in Proc. IEEE/IFIP Int. Symp. Integr. Netw. Manag., May 2001, pp. 609-622.

[21] H.Wang, D. Zhang, and K. G. Shin, "Detecting SYN flooding attacks," in Proc. IEEE INFOCOM, 2002, vol. 3, pp. 1530-1539.

[22] S. Noh, C. Lee, K. Choi, and G. Jung, "Detecting Distributed Denial of Service (DDoS) attacks through inductive learning," Lecture Notes in Computer Science, vol. 2690, pp. 286-295, 2003.https://doi.org/10.1007/978-3-540-45080-1_38. 